\title{
A comparison of an RGB-D camera's performance and a stereo camera in relation to object recognition and spatial position determination
}

\author{
Julian S. Rodriguez* \\ * Ing. Mecatrónica, Universidad de San Buenaventura, Cra 8 H 172-20, Bogotá, Colombia \\ Received 24th of May 2020; Accepted 15th of December 2020
}

\begin{abstract}
Results of using an RGB-D camera (Kinect sensor) and a stereo camera, separately, in order to determine the $3 \mathrm{D}$ real position of characteristic points of a predetermined object in a scene are presented. KAZE algorithm was used to make the recognition, that algorithm exploits the nonlinear scale space through nonlinear diffusion filtering; 3D coordinates of the centroid of a predetermined object were calculated employing the camera calibration information and the depth parameter provided by a Kinect sensor and a stereo camera. Other comparisons have been made using different types of cameras similar to those used in this work, however, a conclusion of the best performance depends on the specific application, for example, it has been shown that for 3D surface reconstruction, the Intel RealSense D415 camera has higher precision than the Kinect.

Experimental results of this work show it is possible to get the required coordinates with both cameras in order to locate a robot, although a balance in the distance where the sensor is placed must be guaranteed: no fewer than $0.8 \mathrm{~m}$ from the object to guarantee the real depth information, it is due to Kinect operating range; $0.5 \mathrm{~m}$ to stereo camera, but it must not be $1 \mathrm{~m}$ away to have a suitable rate of object recognition, however, without loss of generality it can be concluded that the Kinect presents greater precision in the distance measurements with respect to the stereo camera.
\end{abstract}

Stereo camera, 3D coordinates, descriptors, Kinect, image processing, object recognition.

\section{Introduction}

Knowing spatial location of an object in a scene has several applications, particularly, in the field of robotics where visual servoing is usually employed which consists in using visual information obtained from one or more cameras to locate the end effector of a robot in a wanted position [1] and even to follow a trajectory tracking of a movable object [2]. Visual control applications at industrial level have focused on tasks of welding [3] and assembly [4], but there are also important advances in the applications found in other fields as medicine, some examples are presented in [5] and [6].

In literature, visual control diagrams are classified according the camera's location: eye-in-hand camera [7] and eye-to-hand camera [8]; but also in: image-based visual servoing (IBVS) and position-based visual servoing (PBVS) [9], in the first one, the data feedback consists of image characteristics, whereas in the second one information about the actual location of an object is required. In works carried out according PBVS, intrinsic and

Correspondence to: <jrodriguez@usbbog.edu.co $>$

Recommended for acceptance by $<$ Angel D. Sappa $>$

https://doi.org/10.5565/rev/elcvia.1238

ELCVIA ISSN:1577-5097

Published by Computer Vision Center / Universitat Autònoma de Barcelona, Barcelona, Spain 
extrinsic parameters obtained from camera calibration are used to get coordinates in a scene, although depth information must be estimated; other works use stereo calibration employing two cameras in order to get depth information [10].

Recently, depth cameras that use a laser sensor to get information of three dimensions in a scene have been used, the most popular is Microsoft's Kinect which has been employed in investigations in several areas including face recognition [11], 3D scene reconstruction [12] and human-machine interfaces [13]. Employing a sensor that combine RGB camera advantages, this means, the possibility of getting information about morphology and characteristics of an object, with a laser sensor to obtain real information of the depth, allows for determining an object position in the space regarding a coordinate origin without using more than a camera, that allows for getting a system less expensive in terms of economy and computing. Furthermore, there is an emerging market of stereo cameras that use information from two lenses to calculate objects volume and depth employing the epipolar geometry theory [14].

In this paper algorithms of objects recognition in a scene are employed, and the centroid coordinates of the object in order to be used in the object tracking by a robot are obtained. All the process is carried out using two cameras separately: (a Kinect and a stereo camera). The results that were obtained are compared to determine which one is the most suitable to do the previous task according the camera (RGB for Kinect and monochromatic for the stereo) and the technique to calculate the depth of them.

This paper is divided by this way: in the section 2 there is a literature review about the most used descriptors of characteristics to objects recognition, as well as the cameras employed in this work. In the section 3 the used methodology is described through every phase, in this way, the section number 3.2 explains in detail the object recognition in the scene and its segmentation phase; in the 3.3 section the camera information (camera matrix) with the depth information are employed to obtain the real location of a segmented object regarding the image plane; in the 3.4 section the coordinates of a found point regarding the base of the robot serial are shown; in the 4 section the analysis and discussion of results are presented.

\section{State of the art}

Several algorithms have been developed in order to find characteristics of interest within images, those characteristics are generally employed to locate connections between images to obtain similarities between them and to find determinate objects. The most used algorithms are "Scale-invariant feature transform (SIFT" [15],"Center surround extremas for realtime feature detection and matching (CenSurE)" [16] , "Difference of Circles (Doc)" [17] and "Speeded-Up Robust Features (SURF)" [18] and "KAZE"; every one of them has some advantages regarding the amount of similarities that can be found, the time of formulation or invariants in relation to position or scale and other characteristics. In [19] a comparative analysis of different descriptors is carried out where KAZE stands out in several aspects.

Alcantarilla et al. [20] presented KAZE characteristics that exploit a nonlinear scale space through a nonlinear diffusion filtering. That makes the soft focus of images adapt locally to the characteristic points, in this way noise is reduced and at the same time the limits of images areas are preserved. KAZE detector is based on scale normalized determinant of Hessian Matrix (Equation 1), which is calculated in several scale levels.

$$
H_{f}(X)_{i j}=\frac{\partial^{2} f(x)}{\partial x_{i} \partial x_{j}}
$$

Response maximum of the detector are collected as characteristic points using a movable window. The description of characteristic introduces the rotational invariance property when the dominant rotation in a circular neighborhood around every detected characteristic is found. KAZE characteristics are invariant in relation to rotation, scale and limited affinity, and they have a better recognizing in variable scales with a moderate increase in computing time. 
The second equation (Equation 2) shows the classic nonlinear diffusion formulation.

$$
\frac{\partial L}{\partial t}=\operatorname{div}(c(x, y)) \cdot \nabla L
$$

Where "c" is a conductivity function, "div" is divergence, $\nabla$ is gradient operators and "L" is luminance of the image.

Furthermore, different devices have recently been developed which allows for an image acquisition as a conventional camera and obtaining depth information, that permits to get information of 3D objects. As it was told previously, one of the most popular devices is Microsoft's Kinect that employ an infrared projector, as well as an infrared camera to produce an image of depth, additionally, Kinect sensor reproduces video with a frequency of $30 \mathrm{~Hz}$ in RGB 32-bit color and a VGA resolution of $640 X 480$ pixels, QVGA resolution of $620 X 480$ pixels. The limit of Kinect visual range is between 1 and $3.5 \mathrm{~m}$ of distance with a $57^{\circ}$ visual angle horizontally and a $43^{\circ}$ angle vertically [21].

Besides Kinect, stereo cameras have been popularized, with them it is possible to get information of depth through triangulation of the epipolar geometry. Particularly, the camera that was used in this work is an e-Con System Tara which has two monochromatic cameras with resolutions of $752 X 480,640 X 480$ and $320 X 240$ at $60 \mathrm{fps}$ and a distance of base line of $60 \mathrm{~mm}[22]$.

\section{Method}

This paper has two fundamental objectives, the first one is to recognize the position of a specific object in a scene through a camera in real time in order to determine the spatial location of its mass center in relation to the coordinate system of a serial robot; the second one is to compare the results of both kinds of camera that are able to provide 3D information. The Figure 1 shows Phases that are employed to carry out the objective's achievement.

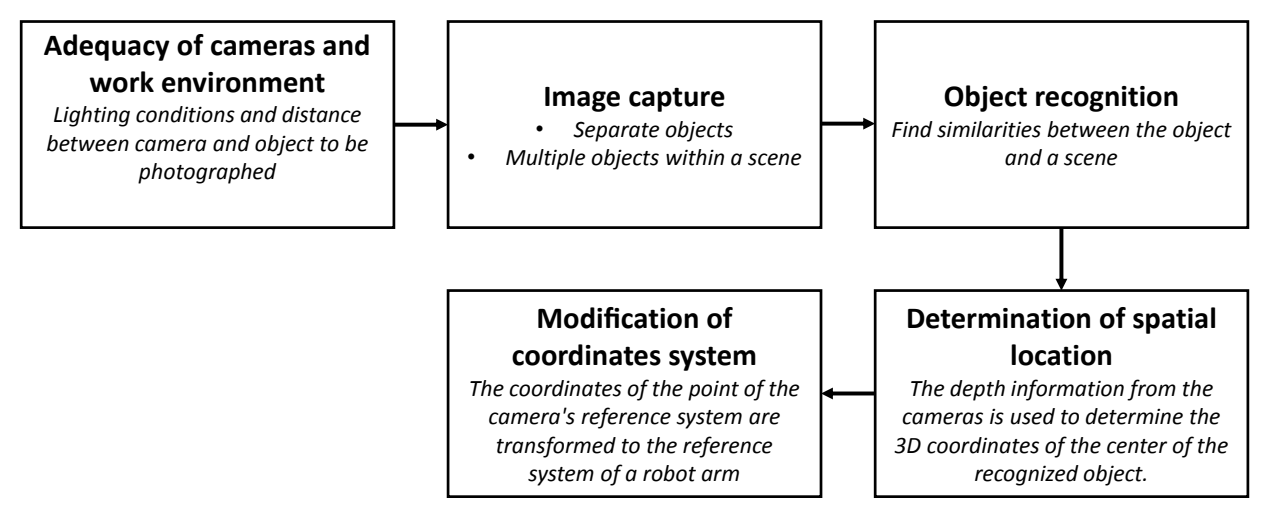

Figure 1: Block diagram of the methodology used. Source: Author

\subsection{Adequacy of cameras and work environment}

Both cameras and objects to be photographed are located at $80 \mathrm{~cm}$ height, the distance between objects and camera could be between $0.5 \mathrm{~m}$ and $1.5 \mathrm{~m}$; moreover, there is just one light source to guarantee that photographs are taken under the same circumstances. Figure 2 shows the described adequacy. 


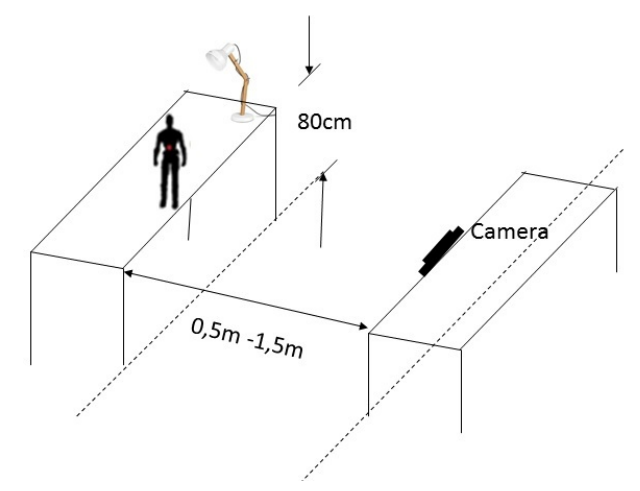

Figure 2: Conditions that were used to take images. Source: Author

\subsection{Object recognition}

Regarding object recognition, literature presents experiments that were carried out according databases which contain 2D and 3D images in several categories, there are some examples in [23] and [24]. In this paper, pictures of objects with similar characteristics are taken and three toy figures were chosen: captain America, Thor and Hulk. In order to do the recognition, image characteristics of every object are extracted separately to search similarities in them and the scene that was registered in real time with every camera.

As it was presented previously, it is possible to obtain the characteristics according several techniques, some of them are Scale-invariant feature transform (SIFT), Center surround extremas for realtime feature detection and matching (CenSurE), Difference of Circles (Doc) y Speeded-Up Robust Features (SURF), although KAZE is used in this work because of the comparative analysis that was carried out in [19] showed that KAZE has a better performance and results in the similarity of characteristics by point.

Following the previous phases in relation to KAZE descriptor, characteristic points of interest that were found separately by the descriptor for each object are presented in figure 3 .

The best bin first [25] was the algorithm used to search similarities, that makes the characteristics of the object coincide with those neighbors of the image which is called scene. The algorithm calculates the distance between vectors of the characteristics in the objects and in the scene to carry out the similarity; the nearest neighbor is defined as the main point with a minimum Euclidean distance to the vector descriptor; in figures 4 and 5 similarities found between both objects and scenes are shown

\subsection{Determination of spatial location}

When the similarities between the characteristics of points of interest in the images of objects and the scene, the result is the object recognition in the set. Spatial location that is wanted to be found is that one of the centroid of polygon which surrounds the object in the scene, there is an example in figure 6 where one of the recognized objects is shown, and also its centroid is highlighted with an asterisk.

Pinhole model describes the geometric relationship between an object coordinates in a 3D space and its projection in an image plane of a camera [26]. Figure 7 illustrates geometric relationships between a point and its projection, those are represented in equation 3. 


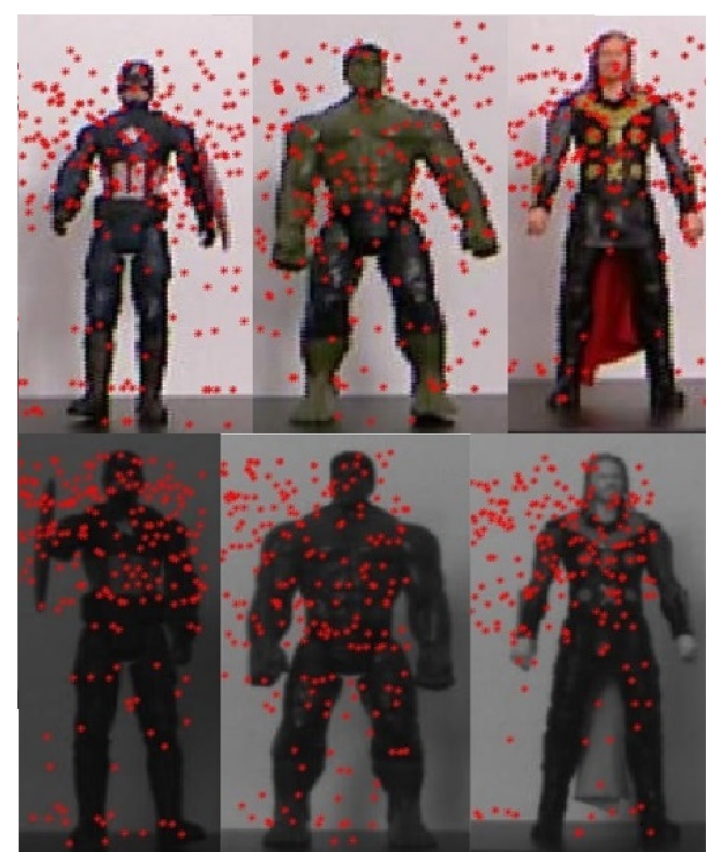

Figure 3: Points of interest detected by KAZE algorithm for each reference object. Top row taken by a Kinect. Lower row taken by a stereo camera. Source: Author

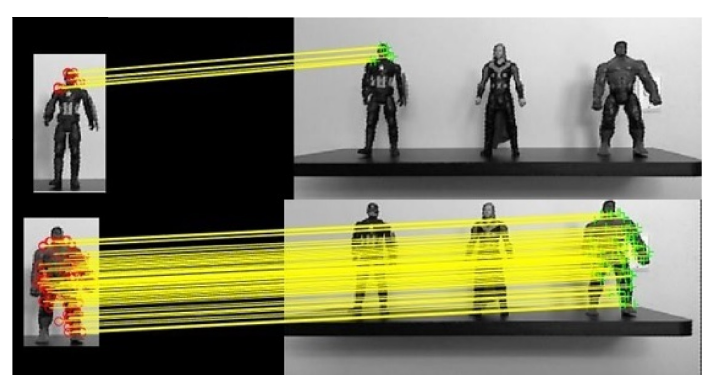

Figure 4: Similarity of points for both objects with Kinect. Source: Author

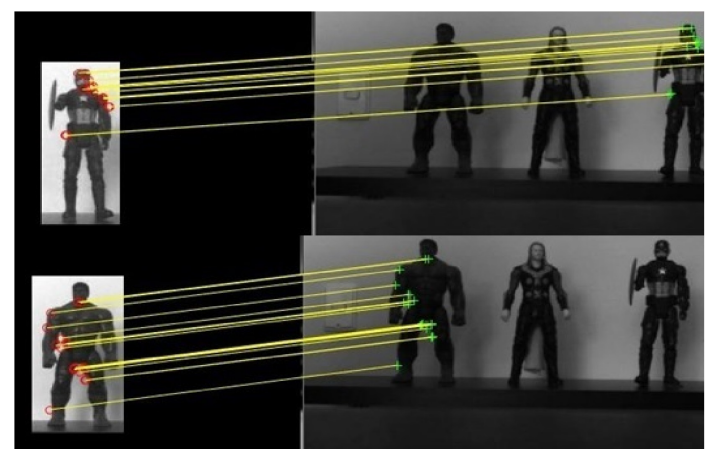

Figure 5: Similarity of points for both objects with stereo camera. Source: Author

$$
\left[\begin{array}{c}
u \\
v \\
1
\end{array}\right]=K\left[\begin{array}{ll}
R & T
\end{array}\right]\left[\begin{array}{c}
X_{w} \\
Y_{w} \\
Z_{w} \\
1
\end{array}\right]
$$




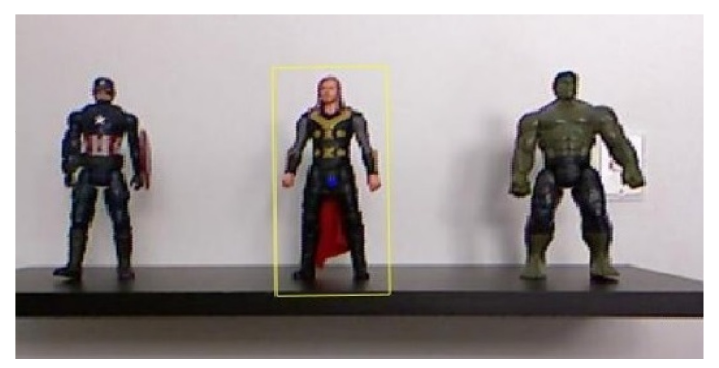

Figure 6: Recognized object in a scene. Source: Author

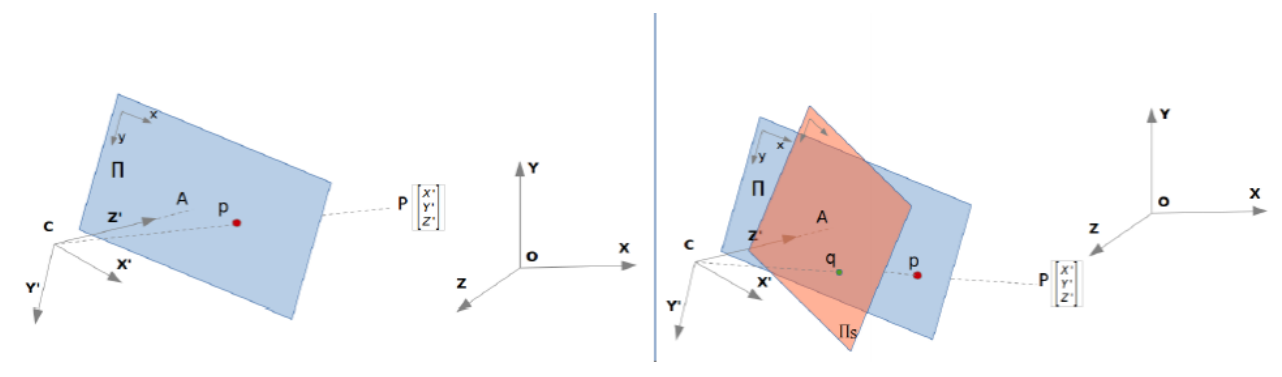

Figure 7: Geometric model of camera. [26]

Where $[u, v, 1]$ and $[X w, Y w, Z w]$ are the homogeneous coordinates of a point in the image and in the 3D scene respectively; $\mathrm{K}$ is the matrix of intrinsic parameters of camera that is composed of the components of focal distance $(f x, f y)$, coordinates of principal point $o^{\prime}(u 0, v 0)$ and distortion factor (s) which are organized according the equation $4 ; R$ and $T$ are rotation and translation matrix to point the scene toward coordinates of the camera known as extrinsic parameters.

$$
K=\left[\begin{array}{ccc}
f x & s & u 0 \\
0 & f y & v 0 \\
0 & 0 & 1
\end{array}\right]
$$

Intrinsic and extrinsic parameters of Kinect camera are found according the calibration procedure described in [27], where an image that works as a pattern with known conditions (usually a chess board) is employed to generate relationships of real world $\left(X_{W}, Y_{W}, Z_{W}\right)$ in meters with coordinates in the image. $\mathrm{K}$ matrix is important during the calibration procedure, regardless correction factor $s$, equation 5 is presented.

$$
K=\left[\begin{array}{ccc}
530.12 & 0 & 239.21 \\
0 & 525.96 & 266.43 \\
0 & 0 & 1
\end{array}\right]
$$

Despite it is possible to estimate the position without having an exact measure of depth, in order to get a later application of positioning, unwanted paths can be generated [28]. For that reason, depth data provided by the selected cameras is employed in this work. Operating range of Kinect is between $1 \mathrm{~m}$ and $3.5 \mathrm{~m}$ while stereo camera's goes from $0.5 \mathrm{~m}$ to $3 \mathrm{~m}$, that limits the possibility of getting a complete spatial information from images which were acquired in those ranges.

Figure 8 shows the depth map generated by Kinect for the scene where one of the objects of search is recognized, as well as a 3D point cloud that is represented in the scene; depth data is taken in the coordinates of polygon centroid that surrounds the known object, in that way with an application of equation 3 it is possible to obtain information about real coordinates of that point location.

Similarly, figure 9 shows the disparity map obtained with stereo camera that allows for getting depth data. 


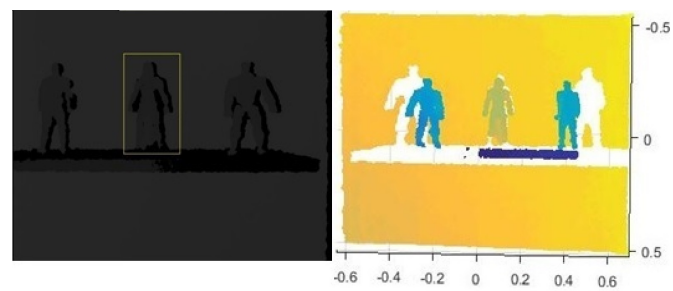

Figure 8: Map of depth of the scene with recognized object. And point cloud obtained with Kinect. Source: Author

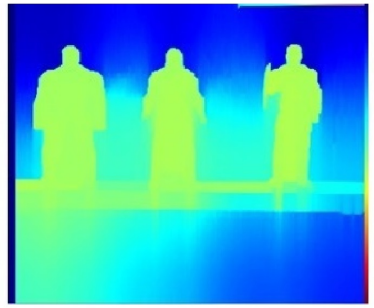

Figure 9: Disparity map obtained with stereo camera. Source: Author

\subsection{Modification of coordinates system}

Both location of the base of a classifier arm and camera location are fixed. In that way, a transformation based in the rotation matrix and the translation vector between systems of coordinates is employed in order to convert the object location of coordinates of the camera into a reference system placed at the base of robot [29]. That converts the point of the camera which was found (shown in the system $(X c, Y c, Z c)$ ) into one that has as reference the system $(X R, Y R, Z R)$ corresponding to the robot. (10)

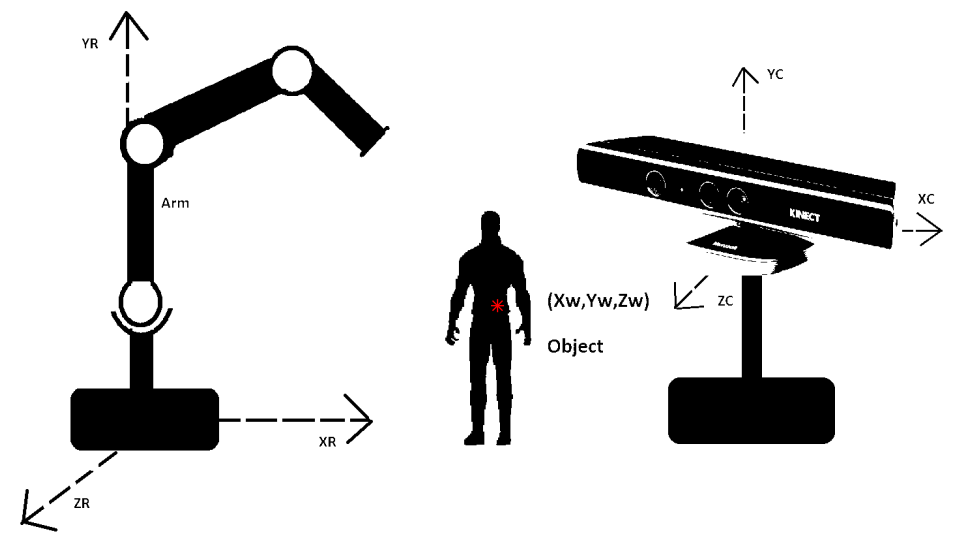

Figure 10: Coordinates systems of camera and robot. Source: Author 


\section{RESULTS AND DISCUSSION}

As it was mentioned previously, this paper objectives are comparing the achievement of both cameras in objects recognition and spatial position. Thus, an experiment was made to valid each objective. The procedure and results are discussed below:

\subsection{Objects recognition}

Images of the same scene form different distances varying objects location and amount were taken. The procedure explained in methodology was applied for each image by calculating characteristic points through KAZE detector in order to find coincidences in the images. Since $1,5 \mathrm{~m}$, images that were taken with any camera have percentages of recognition too low, hence it is not appropriate to recognize objects. That is why distances are modified until $1,5 \mathrm{~m}$. Table 1 sums up the percentages of recognition obtained according the distance of acquisition of Kinect, and in Table 2, there are the same results but with a stereo camera.

Table 1: Results of object recognition in the scene with Kinect. Source: Author

\begin{tabular}{|c|c|c|c|}
\hline Distance/Object & Captain America & Hulk & Thor \\
\hline $0,50 \mathrm{~m}$ & $95 \%$ & $97 \%$ & $90 \%$ \\
\hline $0,7 \mathrm{~m}$ & $90 \%$ & $80 \%$ & $75 \%$ \\
\hline $0,8 \mathrm{~m}$ & $90 \%$ & $80 \%$ & $75 \%$ \\
\hline $1 \mathrm{~m}$ & $86 \%$ & $75 \%$ & $70 \%$ \\
\hline $1,2 \mathrm{~m}$ & $80 \%$ & $70 \%$ & $55 \%$ \\
\hline $1,5 \mathrm{~m}$ & $75 \%$ & $50 \%$ & $45 \%$ \\
\hline
\end{tabular}

Table 2: Results of object recognition in the scene with stereo camera. Source: Author

\begin{tabular}{|c|c|c|c|}
\hline Distance/Object & Captain America & Hulk & Thor \\
\hline $0,50 \mathrm{~m}$ & $95 \%$ & $97 \%$ & $90 \%$ \\
\hline $0,7 \mathrm{~m}$ & $90 \%$ & $80 \%$ & $75 \%$ \\
\hline $0,8 \mathrm{~m}$ & $90 \%$ & $80 \%$ & $75 \%$ \\
\hline $1 \mathrm{~m}$ & $80 \%$ & $75 \%$ & $70 \%$ \\
\hline $1,2 \mathrm{~m}$ & $75 \%$ & $62 \%$ & $50 \%$ \\
\hline $1,5 \mathrm{~m}$ & $40 \%$ & $35 \%$ & $35 \%$ \\
\hline
\end{tabular}

Two main aspects are shown in tables 1 and 2. First, the results of recognition of images that were obtained by the stereo camera are slightly fewer than those obtained by Kinect, which can be attributed to the fact that the images of a stereo camera are slightly darker than those of Kinect, despite photographs are taken with the same resolution and under the same conditions of illumination. Moreover, the percentage of recognition of objects decreases when distance increases with both cameras, that is due to image resolution is low.

After knowing the results of Tables, it is found that images of the scene must be taken at a $0.5 \mathrm{~m}$ distance in order to obtain better results; although, according the objective of this work, that could not be possible with Kinect due to the range of infrared operation starts at $0.8 \mathrm{~m}$, it means, the object would be recognized with a shorter distance, but it is not possible to find real information of depth. 


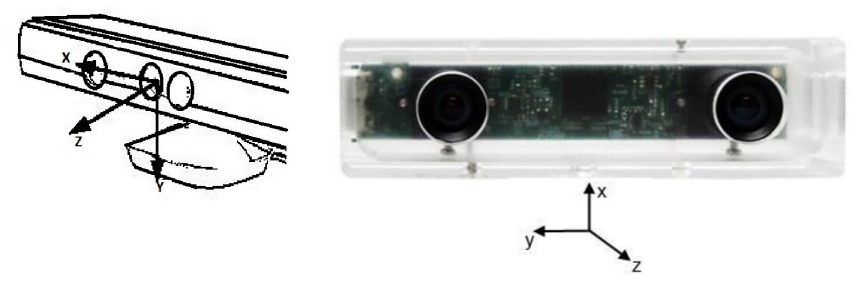

Figure 11: Coordinates systems of Kinect and stereo camera Tara. [22]

\subsection{Validation of objects location}

New images with both devices are taken to valid coordinates that are known and related to the sensor. Centroid coordinates of each object are obtained according to the procedure presented in section 3, and the results are obtained according to the coordinate system presented in Figure 10. Each distance from sensor to the center of the object is validate by measuring centroid location of the object using a measuring tape in order to verify the algorithm results. Results are summed up in Figures 12 and 13 where the vertical axis represents the given measure using a specific camera, and the horizonal axis concerns to the measure found with the measuring tape.

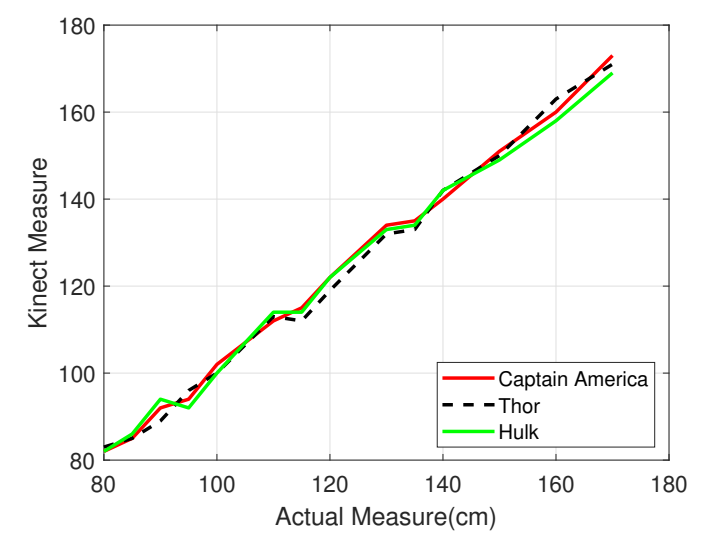

Figure 12: Measures taken with Kinect Vs Distances measured physically. Source: Author

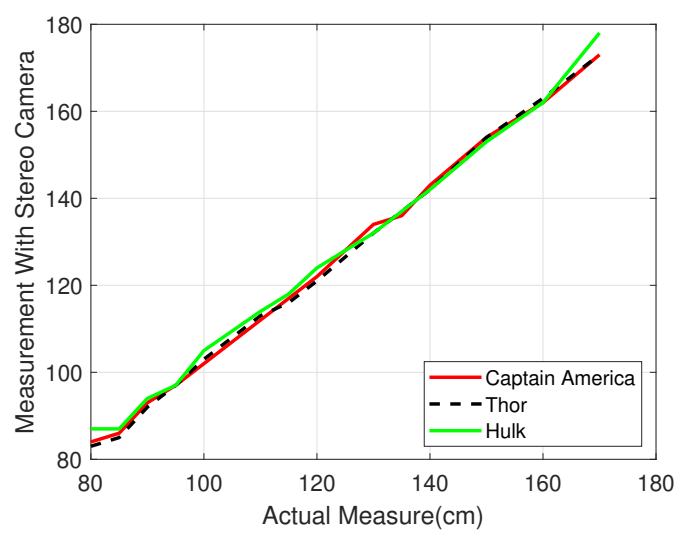

Figure 13: Measures taken with stereo camera Vs Distances measured physically. Source: Author 
Despite differences between the measures of distances of each object with both cameras, the results presented in Figures 12 and 13 show the high precision of the distances data taken with Kinect sensor and the stereo camera, at less for the objective of this work. However, it is possible to deduce that applications where a bigger level of precision as an assembly of little pieces is required, probably a determination of the real location of a robot could not happen due to the differences between the order of millimeters. Moreover, Table 3 allows to have a better depiction of the differences between both devices with a summary of the percentage faults on average of objects distances taken by each camera.

Table 3: Average percentage errors in the distances of each object. Source: Author

\begin{tabular}{|c|c|c|}
\hline Object/Camera & Kinect & Stereo \\
\hline Captain America & $1.2 \%$ & $2.18 \%$ \\
\hline Thor & $1.36 \%$ & $1.88 \%$ \\
\hline Hulk & $1.75 \%$ & $3.19 \%$ \\
\hline General Average & $1.44 \%$ & $2.42 \%$ \\
\hline
\end{tabular}

This Table shows Kinect sensor has less faults with distances measures related to the stereo camera for each object.

After making the experiments presented and noticing there are some differences between the measures of distance for each object with both devices, a third experiment is made without any predetermined object, but by measuring distances to a flat object in order to verify the difference in the result with any dependence on a specific figure. Again, the distances that were obtained are valid with a measuring tape. Figure 14 shows the given results and Table 4 represents the percentage faults on average of the distance from each camera to a flat object.

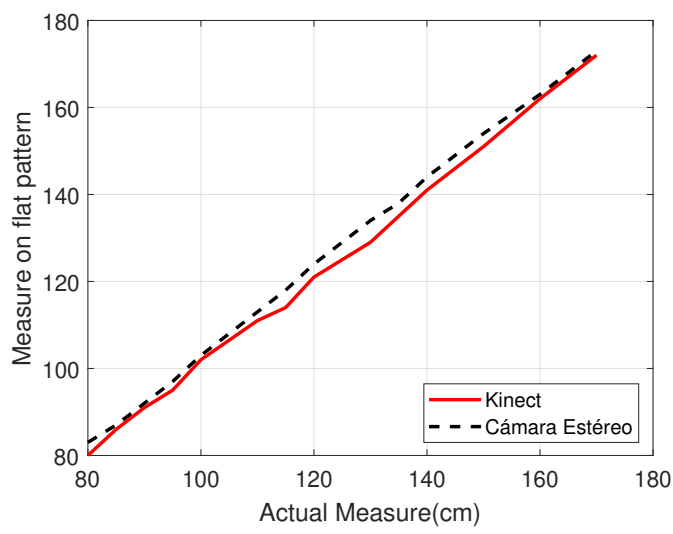

Figure 14: Measures of distance taken on a flat pattern with stereo camera Vs Kinect. Source: Author

Table 4: Average percentage errors of the distance measurement of each camera to a flat object. Source: Author

\begin{tabular}{|c|c|c|}
\hline Object/Camera & Kinect & Stereo \\
\hline Average error percentage & $0.82 \%$ & $2.61 \%$ \\
\hline
\end{tabular}

The response of measures taken on a flat pattern is generally more lineal in relation to measures on other objects for Kinect, that allows for deducing the response in sensor depth depends on reflection surface due to the material or shape. Furthermore, measures taken with the stereo camera are slightly on the real ones, that means Kinect has a better precision with the distance measure regardless the objective of this work. 


\section{Conclusion}

Depth data was employed, as well as the information of image provided by a RGB-D camera (Kinect), and a stereo camera using calibration parameters to determine the real position of the center of an object in a scene, experiments of recognition of objects with similar characteristics in the scene were done applying KAZE algorithm; moreover, the results of depth that was obtained in relation to camera shot were verified.

Images must be taken in short distances (less than $0.5 \mathrm{~m}$ ) to obtain satisfactory results in relation to object recognition due to the low resolution of cameras that were employed. According to those conditions Kinect proved it has a better achievement in the recognition task with characteristic points developed by KAZE algorithm.

Regarding depth data provided by cameras is the right one to achieve the objective of this work, although, the operation ranges of Kinect $(1 m-2.5 m)$ and stereo camera $(0.5 m-3 m)$ do not allow the object to be recognized in the scene in several times despite depth data that was provided by cameras is satisfactory.

Kinect proved a better achievement in the precision of distance measure once again, with an average general fault of $1.44 \%$ instead of $2.42 \%$ given by the stereo camera for the measure of the centroid of predetermined objects, and $0.82 \%$ instead of $2.62 \%$ in the distance to a pattern of flat surface.

Validations of distances show it is possible to obtain coordinates and depth data in order to achieve the wanted application. Although, there is a variation in relation to the distance and the object according the same distances taken in a flat pattern, for that reason, formal research on precision of depth data for Kinect according the reflection surface and other variables carried out in a future work could be something interesting.

\section{References}

[1] Wanbing Zhao et al. "Robust Visual Servoing Control for Ground Target Tracking of Quadrotors". In: IEEE Transactions on Control Systems Technology (2019). DOI: 10.1109/TCST . 2019.2922159.

[2] Xu Jin. "Iterative learning control for non-repetitive trajectory tracking of robot manipulators with joint position constraints and actuator faults". In: International Journal of Adaptive Control and Signal Processing 31.6 (2017), pp. 859-875. DOI: 10 . 1002 / acs . 1098.

[3] Jinquan Li et al. "Structured Light-Based Visual Servoing for Robotic Pipe Welding Pose Optimization". In: IEEE Access 7 (2019), pp. 138327-138340. DOI: 10.1109 / ACCESS . 2019.2943248.

[4] Chicheng Liu et al. "Non-vector space visual servoing for multiple pin-in-hole assembly by robot". In: 2016 IEEE Workshop on Advanced Robotics and its Social Impacts (ARSO). IEEE. 2016, pp. 134-140. DOI: 10.1109 /ARSO.2016.7736270.

[5] Lesley-Ann Duflot et al. "Shearlet-based vs. photometric-based visual servoing for robot-assisted medical applications". In: 2016 IEEE/RSJ International Conference on Intelligent Robots and Systems (IROS). IEEE. 2016, pp. 4099-4104. DOI: 10.1109/IROS .2016.7759603.

[6] Joonho Seo et al. "Ultrasound image based visual servoing for moving target ablation by high intensity focused ultrasound". In: The International Journal of Medical Robotics and Computer Assisted Surgery 13.4 (2017), e1793. DOI: https: / / doi.org/10.1002/rcs.1793.

[7] Mien Van et al. "Fault diagnosis in image-based visual servoing with eye-in-hand configurations using kalman filter". In: IEEE Transactions on Industrial Informatics 12.6 (2016), pp. 1998-2007. DOI: 10 . 1109/TII.2016.2590338. 
[8] Hao Xu, Hesheng Wang, and Weidong Chen. "Uncalibrated visual servoing of mobile manipulators with an eye-to-hand camera". In: 2016 IEEE International Conference on Robotics and Biomimetics (ROBIO). IEEE. 2016, pp. 2145-2150. DOI: 10.1109/ROBIO.2016.7866647.

[9] Josue Lopez-Leyva et al. "Automatic visual servoing control system for industrial robots in arbitrary work areas based on M-PBVS technique". In: Acta Technica Napocensis-Series: Applied Mathematics, Mechanics, and Engineering 62.3 (2019).

[10] Georg R Mueller and Hans-Joachim Wuensche. "Continuous extrinsic online calibration for stereo cameras”. In: 2016 IEEE Intelligent Vehicles Symposium (IV). IEEE. 2016, pp. 966-971. DOI: 10 . 1109 / IVS.2016.7535505.

[11] Billy YL Li et al. "Using kinect for face recognition under varying poses, expressions, illumination and disguise". In: 2013 IEEE workshop on applications of computer vision (WACV). IEEE. 2013, pp. 186192. DOI: $10.1109 /$ WACV. 2013.6475017.

[12] Ming-Der Yang et al. "Image-based 3D scene reconstruction and exploration in augmented reality". In: Automation in Construction 33 (2013), pp. 48-60. DoI: https : / / doi . org/ 10 . 1016 / j . autcon.2012.09.017.

[13] Vinayak Kamath and Sandeep Bhat. "Kinect sensor based real-time robot path planning using hand gesture and clap sound". In: International Conference on Circuits, Communication, Control and Computing. IEEE. 2014, pp. 129-134. DOI: 10.1109/CIMCA.2014. 7057774.

[14] Gang Xu and Zhengyou Zhang. Epipolar geometry in stereo, motion and object recognition: a unified approach. Vol. 6. Springer Science \& Business Media, 2013.

[15] DG Lown. "Distinctive Image Features form Scale-Invariant Keypoint". In: Int'l Journal of Computer Vision 60.2 (2014). DOI: https: / / doi.org/10.1023/B:VISI.0000029664.99615.94.

[16] Agrawal Motilal and Konoligeand MortenRufusBlas Kurt. "CenSurE: Center Surround Extremas for Realtime Feature Detection and Matching". In: Computer Vision - ECCV 2008, 10th European Conference on Computer Vision. Computer Vision - ECCV 2008. 2008, pp. 102-115. DoI: https : / doi . org/ 10.1007/978-3-540-88693-8_8.

[17] A. Hojaij et al. "Difference of Circles Feature Detector". In: 2012 Ninth Conference on Computer and Robot Vision. 2012, pp. 63-69. DOI: 10.1109/CRV.2012.16.

[18] Herbert Bay et al. "Speeded-Up Robust Features (SURF)". In: Computer Vision and Image Understanding 110.3 (2008). Similarity Matching in Computer Vision and Multimedia, pp. 346-359. ISSN: 1077-3142. DoI: https : / / doi .org/10.1016/j . cviu . 2007.09 .014 . URL: http: //www.sciencedirect.com/science/article/pii/s1077314207001555.

[19] Shaharyar Ahmed Khan Tareen and Zahra Saleem. "A comparative analysis of sift, surf, kaze, akaze, orb, and brisk". In: 2018 International conference on computing, mathematics and engineering technologies (iCoMET). IEEE. 2018, pp. 1-10. DOI: 10.1109/ICOMET.2018.8346440.

[20] Pablo Fernández Alcantarilla, Adrien Bartoli, and Andrew J. Davison. "KAZE Features”. In: Computer Vision - ECCV 2012. Ed. by Andrew Fitzgibbon et al. Berlin, Heidelberg: Springer Berlin Heidelberg, 2012, pp. 214-227. ISBN: 978-3-642-33783-3.

[21] Zhengyou Zhang. "Microsoft Kinect Sensor and Its Effect". In: IEEE MultiMedia 19 (Apr. 2012), pp. 412. DOI: 10 . 1109 / MMUL . 2012 . 24. URL: https: / / www . microsoft . com/en-us / research/publication/microsoft-kinect-sensor-and-its-effect/.

[22] e-con Systems India Pvt Ltd. MU Sample Application User. 2014.

[23] Alina Kuznetsova et al. "The open images dataset v4: Unified image classification, object detection, and visual relationship detection at scale". In: arXiv preprint arXiv:1811.00982 (2018). DOI: 10 . 1007 / s11263-020-01316-z. 
[24] Mona Jalal et al. "Sidod: A synthetic image dataset for 3d object pose recognition with distractors". In: Proceedings of the IEEE Conference on Computer Vision and Pattern Recognition Workshops. 2019, pp. 475-477.

[25] Zhenjie Chen and Jingqi Yan. "Fast KNN search for big data with set compression tree and best bin first". In: 2016 2nd International Conference on Cloud Computing and Internet of Things (CCIOT). IEEE. 2016, pp. 97-100. DOI: 10.1109/CCIOT.2016.7868311.

[26] Philippe Cornic et al. "Another look at volume self-calibration: calibration and self-calibration within a pinhole model of Scheimpflug cameras". In: Measurement Science and Technology 27.9 (2016), p. 094004.

[27] Xiaowei Hu et al. "A robust and accurate calibration method for out-of-focus camera". In: Electronic Imaging 2018.2 (2018), pp. 263-1. DOI: https: / / doi .org/ 10 . 2352 / ISSN . 2470-1173. 2018.2 .VIPC-263.

[28] Gabriel J Garcia et al. "Guidance of robot arms using depth data from RGB-D camera". In: 10th International Conference on Informatics in Control, Automation and Robotics (ICINCO 2013). 2013. DOI: $10.5220 / 0004481903150321$.

[29] Bruno Siciliano et al. Robotics. 1st ed. Springer-Verlag London, 2009. ISBN: 978-1-84996-634-4. DOI: $10.1007 / 978-1-84628-642-1$. 